\title{
Symplectic exponentially-fitted modified Runge-Kutta methods of the Gauss type: revisited
}

\author{
G. Vanden Berghe and M. Van Daele \\ Vakgroep Toegepaste Wiskunde en Informatica, Universiteit Gent, \\ Krijgslaan 281 - S9, B - 9000 Gent, Belgium
}

\begin{abstract}
The construction of symmetric and symplectic exponentially-fitted Runge-Kutta methods for the numerical integration of Hamiltonian systems with oscillatory solutions is reconsidered. In previous papers fourth-order and sixth-order symplectic exponentiallyfitted integrators of Gauss type, either with fixed or variable nodes, have been derived. In this paper new such integrators are constructed by making use of the six-step procedure of Ixaru and Vanden Berghe (Exponential fitting, Kluwer Academic Publishers, 2004). Numerical experiments for some oscillatory problems are presented and compared to the results obtained by previous methods.
\end{abstract}

$M S C: 65 \mathrm{~L} 05,65 \mathrm{~L} 06$

Keywords : Exponential fitting, symplecticness, RK-methods, Oscillatory Hamiltonian Systems

\section{Introduction}

The construction of Runge-Kutta (RK) methods for the numerical solution of ODEs, which have periodic or oscillating solutions has been considered extensively in the literature [1]-[12]. In this approach the available information on the solutions is used in order to derive more accurate and/or efficient algorithms than the general purpose algorithms for such type of problems. In [13] a particular six-step flow chart is proposed by which specific exponentiallyfitted algorithms can be constructed. Up to now this procedure has not yet been applied in all its aspects for the construction of symplectic RK methods of Gauss type.

In principle the derivation of exponentially-fitted (EF) RK methods consists in selecting the coefficients of the method such that it integrates exactly all functions of a particular given linear space, i.e. the set of functions

$$
\left\{1, t, \ldots, t^{K}, \exp ( \pm \lambda t), t \exp ( \pm \lambda t), \ldots, t^{P} \exp ( \pm \lambda t)\right\}
$$

where $\lambda \in \mathbb{C}$ is a prescribed frequency. In particular when $\lambda=i \omega, \omega \in \mathbb{R}$ the couple $\exp ( \pm \lambda t)$ is replaced by $\sin (\omega t), \cos (\omega t)$. In all previous papers other set of functions have been introduced.

On the other hand, oscillatory problems arise in different fields of applied sciences such as celestial mechanics, astrophysics, chemistry, molecular dynamics and in many cases the modelling gives rise to Hamiltonian systems. It has been widely recognized by several authors $[8,12],[14]-[16]$ that symplectic integrators have some advantages for the preservation of qualitative properties of the flow over the standard integrators when they are applied to 
Hamiltonian systems. In this sense it may be appropriate to consider symplectic EFRK methods that preserve the structure of the original flow. In [12] the well-known theory of symplectic RK methods is extended to modified (i.e. by introducing additional parameters) EFRK methods, where the set of functions $\{\exp ( \pm \lambda t)\}$ has been introduced, giving sufficient conditions on the coefficients of the method so that symplecticness for general Hamiltonian systems is preserved. Van de Vyver [12] was able to derive a two-stage fourth-order symplectic modified EFRK method of Gauss type. Calvo et al. [2]-[4] have studied two-stage as well as three-stage methods. In their applications they consider pure EFRK methods as well as modified EFRK methods. Their set of functions is the trigonometric polynomial one consisting essentially of the functions $\exp ( \pm \lambda t)$ combined with $\exp ( \pm 2 \lambda t)$ and sometimes $\exp ( \pm 3 \lambda t)$ or a kind of mixed set type where $\exp ( \pm \lambda t)$ is combined with $1, t$ and $t^{2}$. In all cases they constructed fourth-order (two-stage case) and sixth-order (three-stage case) methods of Gauss type with fixed or frequency dependent knot points. On the other hand Vanden Berghe et al. have constructed a two-stage EFRK method of fourth-order integrating the set of functions (1) with $(K=2, P=0)$ and $(K=0, P=1)$, but unfortunately these methods are not symplectic. In addition it has been pointed out in [14] that symmetric methods show a better long time behaviour than non-symmetric ones when applied to reversible differential systems.

In this paper we investigate the construction of two-stage (fourth-order) and three-stage (sixth-order) symmetric and symplectic modified EFRK methods which integrate exactly first-order differential systems whose solutions can be expressed as linear combinations of functions present in the set (1). Our purpose consists in deriving accurate and efficient modified EF geometric integrators based on the combination of the EF approach, followed from the six-step flow chart by Ixaru and Vanden Berghe[13], and symmetry and symplecticness conditions. A sketch of this six-step flow is given in Section 2. The paper is organized as follows. In Section 2 we present the notations and definitions used in the rest of the paper as well as some properties of symplectic and symmetric methods also described in [4]. In Section 3 we derive a class of new two-stage symplectic modified EFRK integrators with frequency dependent nodes and in Section 4 we consider the analogous class of new three-stages method. In Section 5 we present some numerical experiments for sixth-order methods with oscillatory Hamiltonian systems and we compare them with the results obtained by other symplectic (EF)RK Gauss integrators given in $[4,14]$.

\section{Notations and definitions}

We consider initial value problems for first-order differential systems

$$
y^{\prime}(t)=f(t, y(t)), \quad y\left(t_{0}\right)=y_{0} \in \mathbb{R}^{m} .
$$

In case of Hamiltonian systems $m=2 d$ and there exits a scalar Hamiltonian function $H=$ $H(t, y)$, so that $f(y)=-J \nabla_{y} H(t, y)$, where $J$ is the $2 d$-dimensional skew symmetric matrix

$$
J=\left(\begin{array}{cc}
0_{d} & I_{d} \\
-I_{d} & 0_{d}
\end{array}\right), \quad J^{-1}=-J,
$$

and where $\nabla_{y} H(t, y)$ is the column vector of the derivatives of $H(t, y)$ with respect to the components of $y=\left(y_{1}, y_{2}, \ldots, y_{2 d}\right)^{T}$. The Hamiltonian system can then be written as

$$
y^{\prime}(t)=-J \nabla_{y} H(t, y(t)), \quad y\left(t_{0}\right)=y_{0} \in \mathbb{R}^{2 d} .
$$


For each fixed $t_{0}$ the flow map of (2) will be denoted by $\phi_{h}: \mathbb{R}^{m} \rightarrow \mathbb{R}^{m}$ so that $\phi_{h}\left(y_{0}\right)=$ $y\left(t_{0}+h ; t_{0}, y_{0}\right)$. In particular, in the case of Hamiltonian systems, $\phi_{h}$ is a symplectic map for all $h$ in its domain of definition, i.e. the Jacobian matrix of $\phi_{h}\left(y_{0}\right)$ satisfies

$$
\phi_{h}^{\prime}\left(y_{0}\right) J \phi_{h}^{\prime}\left(y_{0}\right)^{T}=J .
$$

A desirable property of a numerical method $\psi_{h}$ for the numerical integration of a Hamiltonian system is to preserve qualitative properties of the original flow $\phi_{h}$ such as the symplecticness, in addition to provide an accurate approximation of the exact $\phi_{h}$.

\section{Definition 2.1}

A numerical method defined by the flow map $\psi_{h}$ is called symplectic if for all Hamiltonian systems (3) it satisfies the condition

$$
\psi_{h}^{\prime}\left(y_{0}\right) J \psi_{h}^{\prime}\left(y_{0}\right)^{T}=J
$$

One of the well-known examples of symplectic numerical methods is the $s$-stage RK Gauss methods which possess order $2 s$. In this paper we shall deal with so-called modified implicit RK-methods, introduced for the first time to obtain explicit EFRK methods [9] and re-used by Van de Vyver [12] for the construction of two-stage symplectic RK methods.

\section{Definition 2.2}

A $s$-stage modified RK method for solving the initial value problems (1) is a one step method defined by

$$
\begin{gathered}
y_{1}=\psi_{h}\left(y_{0}\right)=y_{0}+h \sum_{i=1}^{s} b_{i} f\left(t_{0}+c_{i} h, Y_{i}\right), \\
Y_{i}=\gamma_{i} y_{0}+h \sum_{i=1}^{s} a_{i j} f\left(t_{0}+c_{j} h, Y_{j}\right), \quad i=1, \ldots, s,
\end{gathered}
$$

where the real parameters $c_{i}$ and $b_{i}$ are respectively the nodes and the weights of the method. The parameters $\gamma_{i}$ make the method modified with repect to the classical RK method, where $\gamma_{i}=1, i=1, \ldots, s$. The $s$-stage modified RK-method (5)-(6) can also be represented by means of its Butcher's tableau

$$
\begin{array}{c|c|ccc}
c_{1} & \gamma_{1} & a_{11} & \ldots & a_{1 s} \\
c_{2} & \gamma_{2} & a_{21} & \ldots & a_{2 s} \\
\vdots & \ldots & \vdots & \ddots & \vdots \\
c_{s} & \gamma_{s} & a_{s 1} & \ldots & a_{s s} \\
\hline & & b_{1} & \ldots & b_{s}
\end{array}
$$

or equivalently by the quartet $(c, \gamma, A, b)$.

The conditions for a modified RK method to be symplectic have been obtained by Van de Vyver [12] and they are given in the following theorem.

\section{Theorem 2.3}

A modified RK-method (5)-(6) for solving the Hamiltonian system (3) is symplectic if the following conditions are satisfied

$$
m_{i j} \equiv b_{i} b_{j}-\frac{b_{i}}{\gamma_{i}} a_{i j}-\frac{b_{j}}{\gamma_{j}} a_{j i}=0, \quad 1 \leq i, j \leq s .
$$


In [2] it is shown that a modified RK-method not only preserves the linear invariants but also quadratic invariants if its coefficients satisfy conditions (8).

\section{Definition 2.4}

The adjoint method $\psi_{h}^{*}$ of a numerical method $\psi_{h}$ is the inverse map of the original method with reverse time step $-h$, i.e. $\psi_{h}^{*}:=\psi_{-h}^{-1}$. In other words, $y_{1}=\psi_{h}^{*}\left(y_{0}\right)$ is implicitly defined by $\psi_{-h}\left(y_{1}\right)=y_{0}$. A method for which $\psi_{h}^{*}=\psi_{h}$ is called symmetric.

One of the properties of a symmetric method $\psi_{h}^{*}=\psi_{h}$ is that its accuracy order is even. For $s$-stage modified RK methods whose coefficients are $h$-dependent, as it is the case of EF methods, it is easy to see that the coefficients of $\psi_{h}$ and $\psi_{h}^{*}$ are related by

$c(h)=e-S c^{*}(-h), \quad b(h)=S b^{*}(-h), \quad \gamma(h)=S \gamma^{*}(-h), \quad A(h)=S \gamma^{*}(-h) b^{T}(h)-S A(-h) S$,

where

$$
e=(1, \ldots, 1)^{T} \in \mathbb{R}^{s} \quad \text { and } S=\left(s_{i j}\right) \in \mathbb{R}^{s \times s} \quad \text { with } s_{i j}= \begin{cases}1, & \text { if } i+j=s+1, \\ 0, & \text { if } i+j \neq s+1 .\end{cases}
$$

It has been remarked by Hairer et al. [14] that symmetric numerical methods show a better long time behaviour than nonsymmetric ones when applied to reversible differential equations, as it is the case of conservative mechanical systems. In [3] it is observed that for modified RK methods whose coefficients are even functions of $h$ the symmetry conditions are given by

$$
c(h)+S c(h)=e, \quad b(h)=S b(h), \quad \gamma(h)=S \gamma(h), \quad S A(h)+A(h) S=\gamma(h) b^{T}(h) .
$$

Since for symmetric EFRK methods the coefficients contain only even powers of $h$, the symmetry conditions can be written in a more convenient form by putting [3]

$$
\begin{gathered}
c(h)=\frac{1}{2} e+\theta(h), \quad A(h)=\frac{1}{2} \gamma(h) b^{T}(h)+\Lambda(h), \\
d(h)=\left(\theta_{1}, \ldots, \theta_{s}\right)^{T} \in \mathbb{R}^{s} \text { and } \Lambda=\left(\lambda_{i j}\right) \in \mathbb{R}^{s \times s} .
\end{gathered}
$$

Therefore, for a symmetric EFRK method whose coefficients $a_{i j}$ are defined by

$$
a_{i j}=\frac{1}{2} \gamma_{i} b_{j}+\lambda_{i j}, \quad 1 \leq i, j \leq s,
$$

the symplecticness condtions (8) reduce to

$$
\mu_{i j} \equiv \frac{b_{i}}{\gamma_{i}} \lambda_{i j}+\frac{b_{j}}{\gamma_{j}} \lambda_{j i}=0, \quad 1 \leq i, j, \leq s .
$$

The idea of constructing symplectic EFRK taking into account the six-step procedure [13] is new. We briefly shall survey this procedure and suggest some adaptation in order to make the comparison with previous work more easy.

In step (i) we define the appropriate form of an operator related to the discussed problem. Each of the $s$ internal stages (6) and the final stage (5) can be regarded as being a generalized linear multistep method on a non-uniform grid; we can associated with each of them a linear functional, i.e.

$$
\mathcal{L}_{i}[h, \mathbf{a}] y(t)=y\left(t+c_{i} h\right)-\gamma_{i} y(t)-h \sum_{j=1}^{s} a_{i j} y^{\prime}\left(t+c_{j} h\right), \quad i=1,2, \ldots s .
$$


and

$$
\mathcal{L}[h, \mathbf{b}] y(t)=y(t+h)-y(t)-h \sum_{i=1}^{s} b_{i} y^{\prime}\left(t+c_{i} h\right) .
$$

We further construct the so-called moments which are for Gauss methods the expressions for $L_{i, j}(h, \mathbf{a})=\mathcal{L}_{i}[h, \mathbf{a}] t^{j}, j=0, \ldots, s-1$ and $L_{i}(h, \mathbf{b})=\mathcal{L}[h, \mathbf{b}] t^{j}, j=0, \ldots, 2 s-1$ at $t=0$, respectively.

In step (ii) the linear systems

$$
\begin{gathered}
L_{i j}(h, \mathbf{a})=0, \quad i=1, \ldots, s, \quad j=0,1, \ldots, s-1, \\
L_{i}(h, \mathbf{b})=0, \quad i=0,1, \ldots, 2 s-1 .
\end{gathered}
$$

are solved to reproduce the classical Gauss RK collocation methods, showing the maximum number of functions which can be annihilated by each of the operators.

The steps (iii) and (iv) can be combined in the present context. First of all we have to define all reference sets of $s$ and $2 s$ functions which are appropriate for the internal and final stages respectively. These sets are in general hybrid sets of the following form

$$
\begin{aligned}
& 1, t, t^{2}, \ldots, t^{K} \text { or } t^{K^{\prime}} \\
& \exp ( \pm \lambda t), t \exp ( \pm \lambda t), \ldots, t^{P} \exp ( \pm \lambda t) \text { or } t^{P^{\prime}} \exp ( \pm \lambda t),
\end{aligned}
$$

where for the internal stages $K+2 P=s-3$ and for the final stage $K^{\prime}+2 P^{\prime}=2 s-3$. The set in which there is no classical component is identified by $K=-1$ and $K^{\prime}=-1$, while the set in which there is no exponential fitting component is identified by $P=-1$ or $P^{\prime}=-1$. It is important to note that such reference sets should contain all successive functions inbetween. Lacunary sets are in principle not allowed.

Once the sets chosen the operators (12)-(13) are applied to the members of the sets, in this particular case by taking into account the symmetry and the symplecticness conditions described above. The obtained independent expressions are put to zero and in step (v) the available linear systems are solved. Detailed examples of these technique follow in Sections 3 and 4 . The numerical values for $\lambda_{i j}(h), b_{i}(h), \gamma_{i}(h)$ and $\theta_{i}(h)$ are expressed for real values of $\lambda$ (the pure exponential case) or for pure imaginary $\lambda=i \omega$ (oscillatory case). In order to make the comparison with previous work transparable we have opted to denote the results for real $\lambda$-values.

After the coefficients in the Butcher tableau have been filled in, the principal term of the local truncation error can be written down (step (vi)). Essentially, we know [11] that the algebraic order of the EFRK methods remains the same as the one of the classical Gauss method when this six-step procedure is followed, in other words the algebraic order is $\mathcal{O}\left(h^{2 s}\right)$, while the stage order is $\mathcal{O}\left(h^{s}\right)$. Explicit expressions for this local truncation error will not be discussed here.

\section{Two-stage methods}

In this section we analyze the construction of symmetric and symplectic EFRK Gauss methods with $s=2$ stages whose coefficients are even functions of $h$. These EFRK methods have stage order 2 and algebraic order 4 . From the symmetry conditions (9), taking into account (10) it follows that the nodes $c_{j}=c_{j}(h)$ and weights $b_{j}=b_{j}(h)$ satisfy

$$
c_{1}=\frac{1}{2}-\theta, \quad c_{2}=\frac{1}{2}+\theta, \quad b_{1}=b_{2},
$$


$\theta$ being a real parameter, and the coefficients $a_{i j}=a_{i j}(h)$ and $\gamma_{i}(h)$ satisfy:

$$
a_{11}+a_{22}=\gamma_{1} b_{1}, \quad a_{21}+a_{12}=\gamma_{2} b_{1} .
$$

The symplecticness conditions (8) or (11) are equivalent to

$$
a_{11}=\gamma_{1} b_{1} / 2, \quad \frac{a_{12}}{\gamma_{1}}+\frac{a_{21}}{\gamma_{2}}=b_{1}, \quad a_{22}=\gamma_{2} b_{2} / 2,
$$

which results in

$$
\gamma_{1}=\gamma_{2}, \quad \lambda_{21}=-\lambda_{12}
$$

Taking into account the above relations the Butcher tableau can be expressed in terms of the unknowns $\theta, \gamma_{1}, \lambda_{12}$ and $b_{1}$ :

$$
\begin{array}{c|c|cc}
\frac{1}{2}-\theta & \gamma_{1} & \frac{\gamma_{1} b_{1}}{2} & \frac{\gamma_{1} b_{1}}{2}+\lambda_{12} \\
\frac{1}{2}+\theta & \gamma_{1} & \frac{\gamma_{1} b_{1}}{2}-\lambda_{12} & \frac{\gamma_{1} b_{1}}{2} \\
\hline & & b_{1} & b_{1}
\end{array}
$$

For the internal stages, the relation $K+2 P=-1$ results in the respective $(K, P)$-values:

- $(K=1, P=-1)$ (the classical polynomial case with set $\{1, t\})$, and

- $(K=-1, P=0)$ (the full exponential case with set $\{\exp (\lambda t), \exp (-\lambda t)\})$.

For the outer stage, we have $K^{\prime}+2 P^{\prime}=1$, resulting in the respective $\left(K^{\prime}, P^{\prime}\right)$-values:

- $\left(K^{\prime}=3, P^{\prime}=-1\right)$ (the classical polynomial case with set $\left.\left\{1, t, t^{2}, t^{3}\right\}\right)$,

- $\left(K^{\prime}=1, P^{\prime}=0\right)$ (mixed case with hybrid set $\{1, t, \exp ( \pm \lambda t))$ and

- $\left(K^{\prime}=-1, P^{\prime}=1\right)$ ( the full exponential case with set $\{\exp ( \pm \lambda t), t \exp ( \pm \lambda t)\}$.

The hybrid sets $(K=1, P=-1)$ and $\left(K^{\prime}=3, P^{\prime}=-1\right)$ are related to the polynomial case, giving rise to the well-known RK order conditions and to the fourth order Gauss method [17]

$$
\begin{array}{c|c|cc}
\frac{1}{2}-\frac{\sqrt{3}}{6} & 1 & \frac{1}{4} & \frac{1}{4}-\frac{\sqrt{3}}{6} \\
\frac{1}{2}+\frac{\sqrt{3}}{6} & 1 & \frac{1}{4}+\frac{\sqrt{3}}{6} & \frac{1}{4} \\
\hline & & \frac{1}{2} & \frac{1}{2}
\end{array}
$$

Let us remark that considering the $(K=1, P=-1)$ set for the internal stages gives rise to $\gamma_{1}=1$, a value which is not compatible with the additional symmetry, symplecticity and order conditions imposed. Therefore in what follows we combine the $(K=-1, P=0)$ case with either $\left(K^{\prime}=1, P^{\prime}=0\right)$ or $\left(K^{\prime}=-1, P^{\prime}=1\right)$.

Case $\left(K^{\prime}=1, P^{\prime}=0\right)$

The operators (12) and (13) are applied to the functions present in the occurring hybrid sets, taking into account the structure of the Butcher tableau (14). Following equations arise with 
$z=\lambda h:$

$$
\begin{aligned}
2 b_{1} & =1 \\
2 b_{1} \cosh (z / 2) \cosh (\theta z) & =\frac{\sinh (z)}{z}, \\
\lambda_{12} \cosh (\theta z) & =-\frac{\sinh (\theta z)}{z}, \\
\lambda_{12} \sinh (\theta z)-\frac{\cosh (\theta z)}{z} & =-\frac{\gamma_{1}}{z} \cosh (z / 2),
\end{aligned}
$$

resulting in

$$
\begin{aligned}
& b_{1}=1 / 2, \quad \theta=\frac{\operatorname{arccosh}\left(\frac{2 \sinh (z / 2)}{z}\right)}{z}, \quad \lambda_{12}=-\frac{\sinh (\theta z)}{z \cosh (\theta z)} \\
& \gamma_{1}=\frac{\left(\frac{\sinh (\theta z)^{2}}{z \cosh (\theta z)}+\frac{\cosh (\theta z)}{z}\right) z}{\cosh (z / 2)} .
\end{aligned}
$$

The series expansions for these coefficients for small values of $z$ are given by

$$
\begin{aligned}
\theta & =\sqrt{3}\left(\frac{1}{6}+\frac{1}{2160} z^{2}-\frac{1}{403200} z^{4}+\frac{1}{145152000} z^{6}+\frac{533}{9656672256000} z^{8}-\frac{2599}{2789705318400000} z^{10}+\ldots\right), \\
\lambda_{12} & =\sqrt{3}\left(-\frac{1}{6}+\frac{1}{240} z^{2}-\frac{137}{1209600} z^{4}+\frac{143}{48384000} z^{6}-\frac{81029}{1072963584000} z^{8}+\frac{16036667}{8369115955200000} z^{10}+\ldots\right), \\
\gamma_{1} & =1-\frac{1}{360} z^{4}+\frac{11}{30240} z^{6}-\frac{71}{1814400} z^{8}+\frac{241}{59875200} z^{10}+\ldots,
\end{aligned}
$$

showing that for $z \rightarrow 0$ the classical values are retrieved.

Case $\left(K^{\prime}=-1, P^{\prime}=1\right)$

In this approach equations (16)-(18) remain unchanged and they deliver expressions for $b_{1}, \gamma_{1}$ and $\lambda_{12}$ in terms of $\theta$. Only (15) is replaced by

$$
b_{1}(\cosh (\theta z)(2 \cosh (z / 2)+z \sinh (z / 2))+2 \theta z \cosh (z / 2) \sinh (\theta z))=\cosh (z)
$$

By combining (16) and (19) one obtains an equation in $\theta$ and $z$, i.e.:

$$
\theta \sinh (z) \sinh (\theta z)=\cosh (\theta z)\left(\cosh (z)-\frac{\sinh (z)}{z}-\sinh ^{2}(z / 2)\right) .
$$

It is not anymore possible to write down an analytical solution for $\theta$, but iteratively a series expansion can be derived. We give here those series expansions as obtained for the four unknowns

$$
\begin{aligned}
\theta & =\sqrt{3}\left(\frac{1}{6}+\frac{1}{1080} z^{2}+\frac{13}{2721600} z^{4}-\frac{1}{7776000} z^{6}-\frac{1481}{1810626048000} z^{8}+\frac{573509}{63552974284800000} z^{10}+\ldots\right), \\
b_{1} & =\frac{1}{2}-\frac{1}{8640} z^{4}+\frac{1}{1088640} z^{6}+\frac{1}{44789760} z^{8}-\frac{149}{775982592000} z^{10}+\ldots \\
\lambda_{12} & =\sqrt{3}\left(-\frac{1}{6}+\frac{1}{270} z^{2}-\frac{223}{2721600} z^{4}+\frac{17}{9072000} z^{6}-\frac{259513}{5431878144000} z^{8}+\frac{9791387}{7944121785600000} z^{10}+\ldots\right), \\
\gamma_{1} & =1-\frac{1}{480} z^{4}+\frac{17}{60480} z^{6}-\frac{2629}{87091200} z^{8}+\frac{133603}{43110144000} z^{10}+\ldots
\end{aligned}
$$




\section{Three-stage methods}

The Gauss methods with $s=3$ stages have been analyzed in detail by Calvo et al. [3, 4]. We just shall report here the final results they have obtained by taking into account the symmetry and symplecticity conditions:

$$
\begin{aligned}
c_{1}=\frac{1}{2}-\theta, \quad c_{2} & =\frac{1}{2}, \quad c_{3}=\frac{1}{2}+\theta, \quad b_{3}=b_{1}, \quad \gamma_{3}=\gamma_{1} \\
\Lambda & =\left(\begin{array}{ccc}
0 & -\alpha_{2} & -\alpha_{3} \\
-\alpha_{4} & 0 & \alpha_{4} \\
\alpha_{3} & \alpha_{2} & 0
\end{array}\right)
\end{aligned}
$$

and

$$
\frac{b_{1}}{\gamma_{1}} \alpha_{2}+\frac{b_{2}}{\gamma_{2}} \alpha_{4}=0
$$

The three-stage modified RK-methods are given by the following tableau in terms of the unknowns $\theta, \gamma_{1}, \gamma_{2}, \alpha_{2}, \alpha_{3}, \alpha_{4}, b_{1}$ and $b_{2}$ :

$$
\begin{array}{c|c|ccc}
\frac{1}{2}-\theta & \gamma_{1} & \frac{\gamma_{1} b_{1}}{2} & \frac{\gamma_{1} b_{2}}{2}-\alpha_{2} & \frac{\gamma_{1} b_{1}}{2}-\alpha_{3} \\
\frac{1}{2} & \gamma_{2} & \frac{\gamma_{2} b_{1}}{2}-\alpha_{4} & \frac{\gamma_{2} b_{2}}{2} & \frac{\gamma_{2} b_{1}}{2}+\alpha_{4} \\
\frac{1}{2}+\theta & \gamma_{1} & \frac{\gamma_{1} b_{1}}{2}+\alpha_{3} & \frac{\gamma_{1} b_{2}}{2}+\alpha_{2} & \frac{\gamma_{1} b_{1}}{2} \\
\hline & & b_{1} & b_{2} & b_{1}
\end{array}
$$

For the internal stages the relation $K+2 P=0$ results in the respective $(K, P)$-values:

- $(K=2, P=-1)$ (the classical polynomial case with set $\left.\left\{1, t, t^{2}\right\}\right)$ and

- $(K=0, P=0)$ ( with hybrid set $\{1, \exp ( \pm \lambda t)\})$.

For the final state we have $K^{\prime}+2 P^{\prime}=3$, resulting in the respective $\left(K^{\prime}, P^{\prime}\right)$-values:

- $\left(K^{\prime}=5, P^{\prime}=-1\right)\left(\right.$ the classical polynomial case with set $\left.\left\{1, t, t^{2}, t^{3}, t^{4}, t^{5}\right\}\right)$,

- $\left(K^{\prime}=3, P^{\prime}=0\right)\left(\right.$ with hybrid set $\left.\left\{1, t, t^{2}, t^{3}, \exp ( \pm \lambda t)\right\}\right)$,

- $\left(K^{\prime}=1, P^{\prime}=1\right)($ with hybrid set $\{1, t, \exp ( \pm \lambda t), t \exp ( \pm \lambda t)\})$,

- $\left(K^{\prime}=-1, P^{\prime}=2\right)\left(\right.$ the full exponential case with set $\left.\left\{\exp ( \pm \lambda t), t \exp ( \pm \lambda t), t^{2} \exp ( \pm \lambda t)\right\}\right)$.

The sets $(K=2, P=-1)$ and $\left(K^{\prime}=5, P^{\prime}=-1\right)$ related to the polynomial case gives rise to the order conditions for the three-stage Gauss method of order six [17]

$$
\begin{array}{c|c|ccc}
\frac{1}{2}-\frac{\sqrt{15}}{10} & 1 & \frac{5}{36} & \frac{2}{9}-\frac{\sqrt{15}}{15} & \frac{5}{36}-\frac{\sqrt{15}}{30} \\
\frac{1}{2} & 1 & \frac{5}{36}+\frac{\sqrt{15}}{24} & \frac{2}{9} & \frac{5}{36}-\frac{\sqrt{15}}{24} \\
\frac{1}{2}+\frac{\sqrt{15}}{10} & 1 & \frac{5}{36}+\frac{\sqrt{15}}{30} & \frac{2}{9}+\frac{\sqrt{15}}{15} & \frac{5}{36} \\
\hline & & \frac{5}{18} & \frac{4}{9} & \frac{5}{18}
\end{array}
$$

Following the ideas developed in this paper it should be obvious that we combine the $(K=$ $0, P=0$ ) case with the three non-polynomial cases for the final stage. However keeping 
the 1 in the hybrid set for $(K=0, P=0)$ delivers in $\gamma_{1}=\gamma_{2}=1$, a result which is not compatible with the symplecticity condition (20). Therefore we choose for the internal stages the hybrid set $\{\exp ( \pm \lambda t)\}$, omitting the constant 1; in other words we accept exceptionally a lacunary set, what is principally not allowed by the six-step procedure [13]. Under these conditions, and taking into account the symmetry conditions the $\alpha_{i},(i=2,3,4)$ parameters are the solutions in terms of $\theta, \gamma_{1}$ and $\gamma_{2}$ of the following three equations [4]:

$$
\begin{aligned}
& 1-\gamma_{2} \cosh (z / 2)-2 z \alpha_{4} \sinh (\theta z)=0, \\
& \cosh (\theta z)-\gamma_{1} \cosh (z / 2)+z \alpha_{3} \sinh (\theta z)=0, \\
& \sinh (\theta z)-z \alpha_{3} \cosh (\theta z)-z \alpha_{2}=0
\end{aligned}
$$

thus giving::

$$
\begin{aligned}
& \alpha_{2}=\frac{\cosh (2 \theta z)-\gamma_{1} \cosh (z / 2) \cosh (\theta z)}{z \sinh (\theta z)} \\
& \alpha_{3}=\frac{\gamma_{1} \cosh (z / 2)-\cosh (\theta z)}{z \sinh (\theta z)}, \quad \alpha_{4}=\frac{1-\gamma_{2} \cosh (z / 2)}{2 z \sinh (\theta z)} .
\end{aligned}
$$

For small values of $z$ series expansions are introduced for these expressions (see also next paragraphs). The solution for the other parameters depends essentially on the chosen values of $K^{\prime}$ and $P^{\prime}$.

Case $\left(K^{\prime}=3, P^{\prime}=0\right)$

The operators (12) and (13) are applied to the functions present in the ocurrring hybrid set, taking into account the symmetry conditions; we derive three independent equations in $b_{1}, b_{2}$ and $\theta$, i.e.

$$
\begin{aligned}
2 b_{1}+b_{2} & =1 \\
b_{1} \theta^{2} & =\frac{1}{24}, \\
b_{2}+2 b_{1} \cosh (\theta z) & =\frac{2 \sinh (z / 2)}{z},
\end{aligned}
$$

Taking into account (23) and (25) $b_{1}$ and $b_{2}$ can be expressed in terms of $\theta$ :

$$
b_{1}=\frac{z-2 \sinh (z / 2)}{2 z(1-\cosh (\theta z))}, \quad b_{2}=\frac{2 \sinh (z / 2)-z \cosh (\theta z)}{z(1-\cosh (\theta z))} .
$$

These expressions combined with (24) results in the following equation for $\theta$ :

$$
\theta^{2}-\frac{z(1-\cosh (\theta z))}{12(z-2 \sinh (z / 2))}=0 .
$$

If now the symplecticness condition (20) is imposed, the parameter $\gamma_{1}$ is determined by

$$
\gamma_{1}=\frac{\gamma_{2}(2 \sinh (z / 2)-z) \cosh (2 \theta z)}{2 \sinh (z / 2)-\gamma_{2} \sinh (z)+\left(\gamma_{2} \sinh (z)-z\right) \cosh (\theta z)} .
$$

The obtained parameters define a familiy of EFRK methods which are symmetric and symplectic for all $\gamma_{2} \in \mathbb{R}$. Following [4] we choose from now on $\gamma_{2}=1$.

Now it is easy to give the series expansions for all the coefficients for small values of $z$ : 


$$
\begin{aligned}
\theta= & \sqrt{15}\left(\frac{1}{10}+\frac{1}{21000} z^{2}-\frac{131}{1058400000} z^{4}+\frac{13487}{48898080000000} z^{6}\right. \\
& \left.-\frac{1175117}{3203802201600000000} z^{8}-\frac{505147}{915372057600000000000} z^{10}+\ldots\right) \\
\gamma_{1}= & 1-\frac{3}{70000} z^{6}+\frac{13651}{1176000000} z^{8}-\frac{2452531}{862400000000} z^{10}+\ldots \\
b_{1}= & \frac{5}{18}-\frac{1}{3780} z^{2}+\frac{167}{190512000} z^{4}-\frac{23189}{8801654400000} z^{6}+\frac{7508803}{1153368792576000000} z^{8} \\
& -\frac{87474851}{8073581548032000000000} z^{10}+\ldots \\
b_{2}= & \frac{4}{9}+\frac{1}{1890} z^{2}-\frac{167}{95256000} z^{4}+\frac{23189}{4400827200000} z^{6}-\frac{7508803}{576684396288000000} z^{8} \\
& +\frac{87474851}{4036790774016000000000} z^{10}+\ldots \\
\alpha_{2}= & \sqrt{15}\left(\frac{1}{15}-\frac{1}{6000} z^{2}+\frac{11623}{3175200000} z^{4}-\frac{213648613}{73347120000000} z^{6}+\frac{1669816359863}{2135868134400000000} z^{8}\right. \\
& \left.-\frac{409429160306437}{2135868134400000000000} z^{10}+\ldots\right) \\
\alpha_{3}= & \sqrt{15}\left(\frac{1}{30}+\frac{3}{14000} z^{2}-\frac{24739}{793800000} z^{4}+\frac{14753813}{2993760000000} z^{6}-\frac{7187933379103}{6407604403200000000} z^{8}\right. \\
& \left.+\frac{48242846122937}{177989011200000000000} z^{10}+\ldots\right) \\
\alpha_{4}= & \sqrt{15}\left(-\frac{1}{24}+\frac{13}{67200} z^{2}-\frac{37}{12700800} z^{4}+\frac{19922401}{469421568000000} z^{6}-\frac{733072729}{1220496076800000000} z^{8}\right. \\
& \left.+\frac{1539941201}{183074411520000000000} z^{10}+\ldots\right) .
\end{aligned}
$$

Case $\left(K^{\prime}=1, P^{\prime}=1\right)$

The equations (23) and (25) remain unchanged. Equation (24) is replaced by the equation obtained by applying the operator (13) with $s=3$ on $t \exp ( \pm \lambda t)$ resulting in:

$$
2 b_{1} z^{2} \theta \sinh (\theta z)=z \cosh (z / 2)-2 \sinh (z / 2) .
$$

Taking into account (25) and (26) $b_{1}$ and $b_{2}$ can be expressed in terms of $\theta$ :

$$
\begin{aligned}
& b_{1}=\frac{z \cosh (z / 2)-2 \sinh (z / 2)}{2 z^{2} \theta \sinh (\theta z)} \\
& b_{2}=\frac{-\cosh (\theta z) z \cosh (z / 2)+2 \cosh (\theta z) \sinh (z / 2)+2 \sinh (z / 2) z \theta \sinh (\theta z)}{z^{2} \theta \sinh (\theta z)}
\end{aligned}
$$

Introducing these results for $b_{1}$ and $b_{2}$ into (23) provides an equation for $\theta$ :

$$
\frac{(1-\cosh (\theta z))(z \cosh (z / 2)-2 \sinh (z / 2))+z \theta \sinh (\theta z)(2 \sinh (z / 2)-z)}{z^{2} \theta \sinh (\theta z)}=0 .
$$

From the symplecticness condition (20) an expression for $\gamma_{1}$ follows:

$$
\gamma_{1}=\frac{\gamma_{2} \cosh (2 \theta z)(z \cosh (z / 2)-2 \sinh (z / 2))}{\cosh (\theta z)(z \cosh (z / 2)-2 \sinh (z / 2))-2 \sinh (z / 2) z \theta \sinh (\theta z)\left(1-\gamma_{2} \cosh (z / 2)\right)} .
$$


Again we choose $\gamma_{2}=1$. The series expansions for the different parameters now follow immediately:

$$
\begin{aligned}
& \theta=\sqrt{15}\left(\frac{1}{10}+\frac{1}{10500} z^{2}-\frac{31}{117600000} z^{4}+\frac{2869}{5433120000000} z^{6}-\frac{332933}{355978022400000000} z^{8}\right. \\
& \left.+\frac{1792783}{711956044800000000000} z^{10}+\ldots\right) \\
& \gamma_{1}=1-\frac{9}{280000} z^{6}+\frac{6861}{784000000} z^{8}-\frac{3685091}{1724800000000} z^{10}+\ldots \\
& b_{1}=\frac{5}{18}-\frac{1}{1890} z^{2}-\frac{23}{21168000} z^{4}+\frac{3383}{244490400000} z^{6}-\frac{6186473}{128152088064000000} z^{8} \\
& +\frac{6259951}{448532308224000000000} z^{10}+\ldots \\
& b_{2}=\frac{4}{9}+\frac{1}{945} z^{2}+\frac{23}{10584000} z^{4}-\frac{3383}{122245200000} z^{6}+\frac{6186473}{64076044032000000} z^{8} \\
& -\frac{6259951}{224266154112000000000} z^{10}+\ldots \\
& \alpha_{2}=\sqrt{15}\left(\frac{1}{15}-\frac{1}{18000} z^{2}+\frac{1063}{352800000} z^{4}-\frac{4445759}{2037420000000} z^{6}+\frac{1250913246151}{2135868134400000000} z^{8}\right. \\
& \left.-\frac{305480839860709}{2135868134400000000000} z^{10}+\ldots\right) \\
& \alpha_{3}=\sqrt{15}\left(\frac{1}{30}+\frac{19}{126000} z^{2}-\frac{2179}{88200000} z^{4}+\frac{8735197}{2328480000000} z^{6}-\frac{1798803442789}{2135868134400000000} z^{8}\right. \\
& \left.+\frac{216068604952379}{1067934067200000000000} z^{10}+\ldots\right) \\
& \alpha_{4}=\sqrt{15}\left(-\frac{1}{24}+\frac{43}{201600} z^{2}-\frac{59}{28224000} z^{4}+\frac{1419377}{52157952000000} z^{6}-\frac{431537179}{1220496076800000000} z^{8}\right. \\
& \left.+\frac{237023071}{53396703360000000000} z^{10}+\ldots\right) \text {. }
\end{aligned}
$$

Case $\left(K^{\prime}=-1, P^{\prime}=2\right)$

The equations (25) and (26) remain unchanged. A third equation is added which follows from the application of the operator (13) with $s=3$ on $t^{2} \exp ( \pm \lambda t)$, i.e.:

$$
\begin{aligned}
& b_{1} \cosh (z \theta)\left(2 \cosh (z / 2)+\frac{1}{2} z \sinh (z / 2)+2 z \theta^{2} \sinh (z / 2)\right)-\cosh (z) \\
& +2 b_{1} \sinh (z \theta)(2 \theta \sinh (z / 2)+z \theta \cosh (z / 2))+b_{2}\left(\cosh (z / 2)+\frac{1}{4} z \sinh (z / 2)\right)=0(30)
\end{aligned}
$$

The formal expression for $b_{1}$ and $b_{2}$ remain respectively (27) and (28). Introducing these expression for $b_{1}$ and $b_{2}$ into (30) gives us an equation for $\theta$. From the symplecticness condition (20) again the expression (29) for $\gamma_{1}$ follows. Again by chosing $\gamma_{2}=1$, the series expansion of the different parameters follow: 


$$
\begin{aligned}
\theta= & \sqrt{15}\left(\frac{1}{10}+\frac{1}{7000} z^{2}-\frac{37}{88200000} z^{4}-\frac{2323}{679140000000} z^{6}+\frac{466717}{8899450560000000} z^{8}\right. \\
& \left.-\frac{15014807}{66745879200000000000} z^{10}+\ldots\right) \\
\gamma_{1}= & 1-\frac{27}{1120000} z^{6}+\frac{41379}{6272000000} z^{8}-\frac{22149861}{13798400000000} z^{10}+\ldots \\
b_{1}= & \frac{5}{18}-\frac{1}{1260} z^{2}-\frac{187}{31752000} z^{4}+\frac{11887}{91683900000} z^{6}-\frac{14932867}{16019011008000000} z^{8} \\
& \quad-\frac{16262011}{28033269264000000000} z^{10}+\ldots \\
b_{2}= & \frac{4}{9}+\frac{1}{630} z^{2}+\frac{187}{15876000} z^{4}+\frac{173633}{733471200000} z^{6}-\frac{52835987}{32038022016000000} z^{8} \\
& +\frac{817009801}{224266154112000000000} z^{10}+\ldots \\
\alpha_{2}= & \sqrt{15}\left(\frac{1}{15}+\frac{1}{18000} z^{2}+\frac{719}{176400000} z^{4}-\frac{157253603}{97796160000000} z^{6}+\frac{468408965117}{1067934067200000000} z^{8}\right. \\
& \left.-\frac{76002203332597}{711956044800000000000} z^{10}+\ldots\right) \\
\alpha_{3}= & \sqrt{15}\left(\frac{1}{30}+\frac{11}{126000} z^{2}-\frac{3523}{176400000} z^{4}+\frac{40063763}{13970880000000} z^{6}-\frac{675385487507}{1067934067200000000} z^{8}\right. \\
& \left.+\frac{322656693230117}{2135868134400000000000} z^{10}+\ldots\right) \\
\alpha_{4}= & \sqrt{15}\left(-\frac{1}{24}+\frac{47}{201600} z^{2}-\frac{73}{56448000} z^{4}+\frac{1520789}{156473856000000} z^{6}-\frac{220181869}{1220496076800000000} z^{8}+\right. \\
& \left.\frac{47152907}{14063329280000000000} z^{10}+\ldots\right) . \\
= &
\end{aligned}
$$

\section{Remark:}

Sixth-order symmetric and symplectic modified Runge-Kutta methods of Gauss type have been contructed by others. In [3] the authors constructed such methods by making use of a basic set consisting of $\{\exp ( \pm \lambda t), \exp ( \pm 2 \lambda t), \exp ( \pm 3 \lambda t)\}$ with fixed $\theta$-values and frequency dependent $\theta$-values. In [4] analogous constructions are discussed based on a reference set $\left\{t, t^{2}, \exp ( \pm \lambda t)\right\}$, again with fixed and frequency dependent $\theta$-values. In both cases the results are in a sense comparable with ours and in the numerical experiments we shall compare the results of $[4]$ with the ones we have obtained.

\section{$5 \quad$ Numerical experiments}

In this section we report on some numerical experiments where we test the effectiveness of the new and the previous [4] modified Runge-Kutta methods when they are applied to the numerical solution of several differential systems. All the considered codes have the same qualitative properties for the Hamiltonian systems. In the figures we show the decimal logarithm of the maximum global error versus the number of steps required by each code in logarithmic scale. All computations were carried out in double precision and series expansions are used for the coefficients when $|z|<0.1$. In all further displayed figures following results 
are shown: the method of Calvo et al. with constant nodes (const) and with variable nodes (var), the classical Gauss results (class) and the results obtained with the new methods with $P=0(\mathrm{P} 0), P=1(\mathrm{P} 1)$ and $P=2(\mathrm{P} 2)$.

Problem 1: Kepler's plane problem defined by the Hamiltonian function

$$
H(p, q)=\frac{1}{2}\left(p_{1}^{2}+p_{2}^{2}\right)-\left(q_{1}^{2}+q_{2}^{2}\right)^{-1 / 2},
$$

with the initial conditions $q_{1}(0)=1-e, q_{2}(0)=0, p_{1}(0)=0, p_{2}(0)=((1+e) /(1-e))^{\frac{1}{2}}$, where $e,(0 \leq e<1)$ represents the eccentricity of the elliptic orbit. The exact solution of this IVP is a $2 \pi$-periodoc elliptic orbit in the $\left(q_{1}, q_{2}\right)$-plane with semimajor axis 1 , corresponding the starting point to the pericenter of this orbit. In the numerical experiments presented here we have chosen the same values as in [4], i.e. $e=0.001, \lambda=i \omega$ with $\omega=\left(q_{1}^{2}+q_{2}^{2}\right)^{-\frac{3}{2}}$ and the integration is carried out on the interval $[0,1000]$ with the steps $h=1 / 2^{m}, m=1, \ldots, 4$. The numerical behaviour of the global error in the solution is presented in figure 1 . The results obtained by the three new constructed methods are falling together. One cannot distinguish the results. They are comparable to the ones obtained by Calvo and more accurate than the results of the classical Gauss method of the same order. Remark that $e$ has been kept small as it was the case in previous papers. We have however observed that increasing $e$ does not changed the conclusions reached.
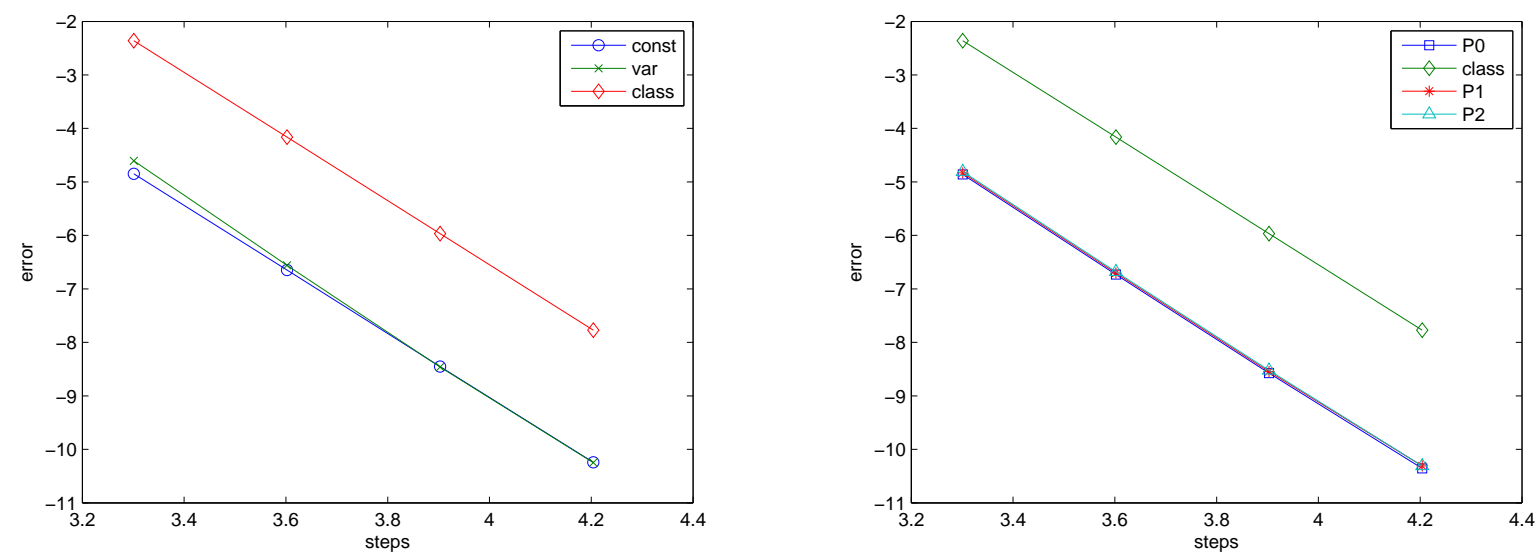

Figure 1: Maximum global error in the solution of Problem 1. In the left figure the results obtained by the methods of Calvo et al. [4] are displayed. In the right figure the results obtained with the methods of order six derived in this paper are shown.

Problem 2 A perturbed Kepler's problem defined by the Hamiltonian function

$$
H(p, q)=\frac{1}{2}\left(p_{1}^{2}+p_{2}^{2}\right)-\frac{1}{\left(q_{1}^{2}+q_{2}^{2}\right)^{1 / 2}}-\frac{2 \epsilon+\epsilon^{2}}{3\left(q_{1}^{2}+q_{2}^{2}\right)^{3 / 2}},
$$

with the initial conditions $q_{1}(0)=1, q_{2}(0)=0, p_{1}(0)=0, p_{2}(0)=1+\epsilon$, where $\epsilon$ is a small positive parameter. The exact solution of this IVP is given by

$$
q_{1}(t)=\cos (t+\epsilon t), \quad q_{2}(t)=\sin (t+\epsilon t), \quad p_{i}(t)=q_{i}^{\prime}(t), i=1,2 .
$$


As in [4] the numerical results are computed with the integration steps $h=1 / 2^{m}, m=$ $1, \ldots, 4$. We take the parameter $\epsilon=10^{-3}, \lambda=i \omega$ with $\omega=1$ and the problem is integrated up to $t_{\text {end }}=1000$. . The global error in the solution is presented in figure 2. For our methods we have the same conclusions as for the Problem 1. On the contrary for the results of Calvo the results obtained with fixed $\theta$-values are more accurate than the ones obtained by variable $\theta$-values.
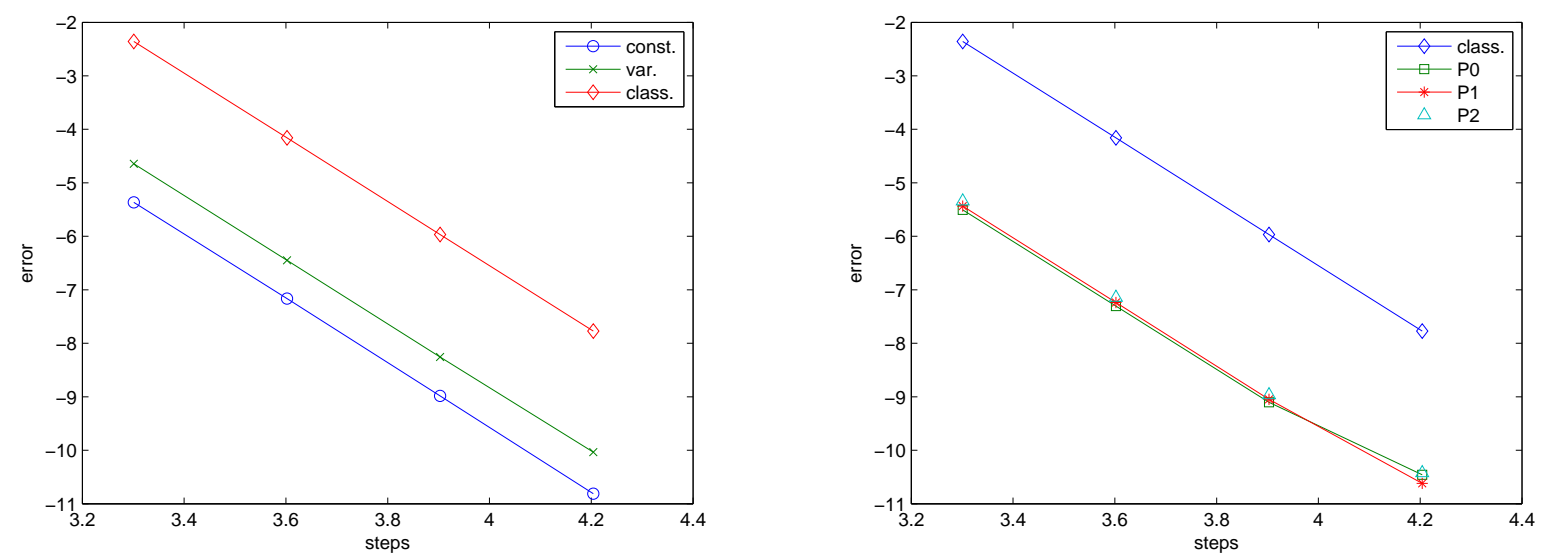

Figure 2: Maximum global error in the solution of Problem 2. In the left figure the results obtained by the methods of Calvo et al. [4] are displayed. In the right figure the results obtained with the methods of order six derived in this paper are shown.

Problem 3 Euler's equations that describe the motion of a rigid body under no forces

$$
\dot{q}=f(q)=\left((\alpha-\beta) q_{2} q_{3},(1-\alpha) q_{3} q_{1},(\beta-1) q_{1} q_{2}\right)^{T},
$$

with the initial values $q(0)=(0,1,1)^{T}$, and the parameter values $\alpha=1+\frac{1}{\sqrt{1.51}}$ and $\beta=1-\frac{0.51}{\sqrt{1.51}}$. The exact solution of this IVP is given by

$$
q(t)=(\sqrt{1.51} \operatorname{sn}(t, 0.51), \operatorname{cn}(t, 0.51), \operatorname{dn}(t, 0.51))^{T},
$$

it is periodic with period $T=7.45056320933095$, and sn, cn, dn stand for the elliptic Jacobi functions. Figure 3 shows the numerical results obtained for the global error computed with the interation steps $h=1 / 2^{m}, m=1, \ldots, 4$, on the interval $[0,1000]$, and $\lambda=i 2 \pi / T$. The results of Calvo et al are all of the same accuracy while in our approach the EF methods are still more accurate than the classical one. In this problem the choice of the frequency is not so obvious and therefore the differentiation between the classical and the EF methods is not so pronounced.

\section{Conclusions}

In this paper another approach for constructing symmetric symplectic modified EFRK methods based upon the sixth-step procedure of [13] is presented. Two-stage fourth-order and 

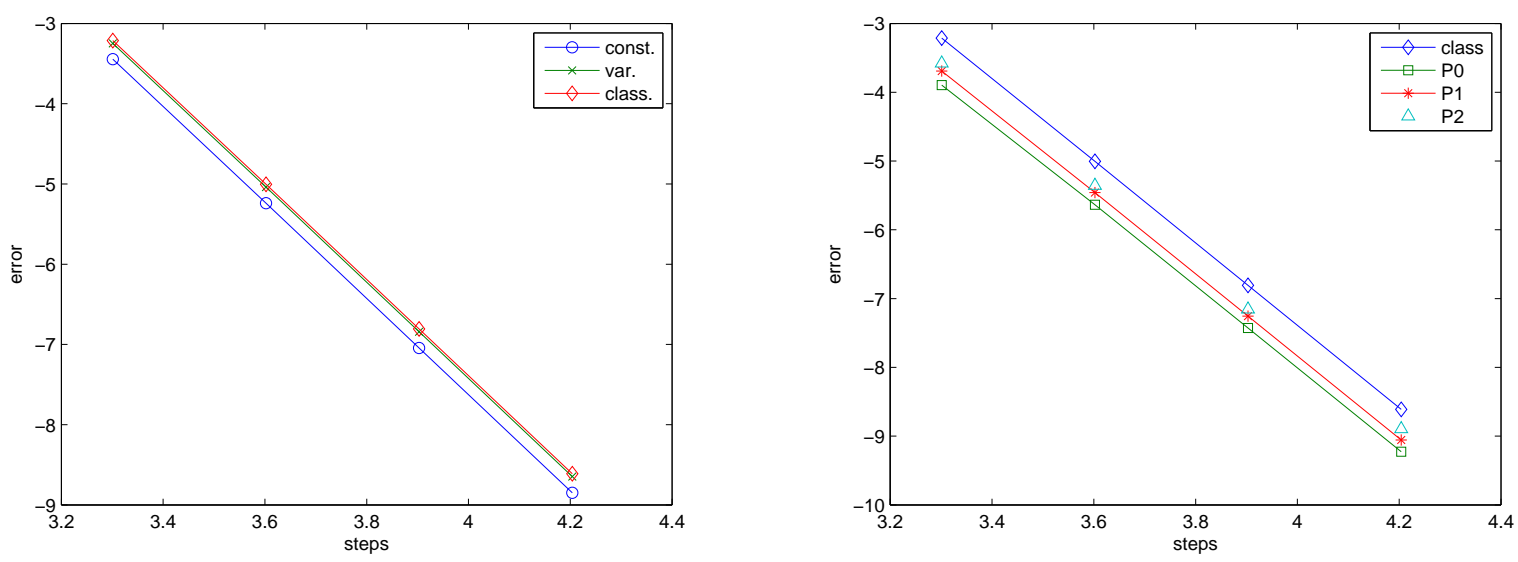

Figure 3: Maximum global error in the solution of Problem 3. In the left figure the results obtained by the methods of Calvo et al. [4] are displayed. In the right figure the results obtained with the methods of order six derived in this paper are shown.

three-stage sixth-order integrators of Gauss type which are symmetric and symplectic and which preserve linear and quadratic invariants have been derived. When the frequency used in the exponential fitting process is put to zero all considered integrators reduce to the classical Gauss integrator of the same order. Some numerical experiments show the utility of these new integrators for some oscillatory problems. The results obtained here are quite similar to the ones obtained in [4], but they differ in some of the details. The introduced method can be extended to EFRK with larger algebraic order.

\section{References}

[1] D. G. Bettis, Runge-Kutta algorithms for oscillatory problems, J. Appl. Math. Phys. (ZAMP) 30 (1979) 699-704.

[2] M. Calvo, J. M. Franco, J. I. Montijano, L. Rández, Structure preservation of exponentially fitted Runge-Kutta methods, Journ. Comp. Appl. Math. 218 (2008) 421-434.

[3] M. Calvo, J. M. Franco, J. I. Montijano, L. Rández, Sixth-order symmetric and symplectic exponentially fitted Runge-Kutta methods of the Gauss type, Comp. Phys. Commun. 178 (2008) 732-744.

[4] M. Calvo, J. M. Franco, J. I. Montijano, L. Rández, Sixth-order symmetric and symplectic exponentially fitted modified Runge-Kutta methods of the Gauss type, Journ. Comp. Appl. Math. 223 (2009) 387-398 .

[5] J. M. Franco, Runge-Kutta methods adapted to the numerical integration of oscillatory problems, Appl. Numer. Math. 50 (2004) 427-443.

[6] K. Ozawa, A functional fitting Runge-Kutta method with variable coefficients, Japan J. Indust. Appl. Math. 18 (2001) 107-130.

[7] T. E. Simos, An exponentially-fitted Runge-Kutta method for the numerical integration of initial-value problems with periodic or oscillating solutions, Comp. Phys. Commun. 115 (1998) 1-8. 
[8] T. E. Simos, J. Vigo-Aguiar, Exponentially-fitted symplectic integrator, Phys. Rev. E67 (2003)1-7.

[9] G. Vanden Berghe, H. De Meyer, M. Van Daele, T. Van Hecke, Exponentially-fitted explicit Runge-Kutta methods, Comp. Phys. Commun. 123 (1999) 7-15.

[10] G. Vanden Berghe, H. De Meyer, M. Van Daele, T. Van Hecke, Exponentially-fitted Runge-Kutta methods, Journ. Comp. Appl. Math. 125 (2000)107-115.

[11] G. Vanden Berghe, M. Van Daele, H. Van de Vyver, Exponentially-fitted RungeKutta methods of collocation type: Fixed or variable knot points?, Journ. Comp. Appl. Math. 159 (2003) 217-239.

[12] H. Van de Vyver, A fourth order symplectic exponentially fitted integrator, Comp. Phys. Commun. 174 (2006) 255-262.

[13] L. Gr. Ixaru, G. Vanden Berghe, Exponential Fitting, Mathematics and its applications vol. 568, Kluwer Academic Publishers, 2004.

[14] E. Hairer, C. Lubich, G. Wanner, Geometric Numerical Integration: Structure Preserving Algorithms for Ordinary Differential Equations, Springer Verlag, Berlin 2002.

[15] J. M. Sanz-Serna, Symplectic integrators for Hamiltonian problems: An overview, Acta Numerica 1 (1992) 243-286.

[16] J. M. Sanz-Serna, M. P. Calvo, Numerical Hamiltonian Problems, Chapman and Hall, London 1994.

[17] E. Hairer, S. P. Nørsett, G. Wanner, Solving Ordinary Differential Equations I, Nonstiff Problems, Springer-Verlag Berlin, Heidelberg 1993. 